\title{
Lehrlingsaustauschprojekt für Medizinische Praxisassistentinnen
}

\section{Gantner}

* Die 1999 gegründete Margrit und Ruth Stellmacher-Stiftung ist statutengemäss der Unterstützung von Medizinischen Praxisassistentinnen verpflichtet, sei es zur Unterstützung der Ausbildung oder als Hilfe in finanzieller Not.

Korrespondenzen:

Dr. med. Verena Gantner Kirchbühlstrasse 13

CH-5630 Muri

Margrit und

Ruth Stellmacher-Stiftung

Dr. iur. Thomas Brender

Bahnhofstrasse 61

Postfach 7675

CH-8023 Zürich

Tel. 0442258888

\section{Grundidee}

Jugendliche Lernende profitieren erfahrungsgemäss viel von einem Austausch oder Auslandaufenthalt. Dieses bei Studenten seit langem rege benutzte Instrument zur Horizonterweiterung möchten wir auch jugendlichen Lernenden, im speziellen medizinischen Praxisassistentinnen, ermöglichen.

Wenige Wochen in einer andern Arztpraxis, eventuell in einem andern Land oder einer andern Sprachregion zu arbeiten und idealerweise auch zur Schule gehen zu können und dort Lehrmeister, Lehrlinge und eine andere Region kennenzulernen ist eine spezielle Erfahrung. Der Profit eines derartigen Austauschs liegt aber selbstverständlich nicht ausschliesslich im fachlichen Bereich. Die meisten Lehrlinge betonen vor allem den persönlichen Profit. Die Förderung der Selbständigkeit, Selbstverantwortung und des Selbstwertgefühls beeinflusst und fördert den Reifungsprozess der jungen Lernenden.

Konsultative Umfragen an MPA-Schulen in den Kantonen Bern und Aargau haben ein grosses Interesse der Lernenden für ein Austauschprogramm erbracht.

\section{Organisation}

Sinnvollerweise umfasst ein Austauschpraktikum 2-4 Wochen und die Zeiten werden individuell vereinbart, so dass möglichst wenige Schulstunden verpasst werden. Ein Austausch ganz zu Beginn der Lehre oder kurz vor der Lehrabschlussprüfung ist ungünstig. Am besten eignet sich die Zeit zwischen dem 3. und 5. Semester.

Am besten sollte ein Teil der Austauschzeit in die Schulferien fallen. Natürlich wird aber die übliche 5wöchige Ferienzeit der Lernenden nicht beschnitten. Mehr Informationen finden sich auf der Homepage von Visite unter www.visite.ch.

Die Austauschorganisation «Visite» gehört zur Rotariervereinigung und hat seit einigen Jahren Erfahrungen im Austausch von Lehrlingen unterschiedlichster Berufe. An jährlichen Treffen werden Erfahrungen ausgetauscht und
Une initiative de la Fondation

Margrit et Ruth Stellmacher: projet d'échange d'apprentis et d'apprenties au cours de la formation des assistantes médicales

Afin d'élargir l'expérience professionnelle et personnelle des apprenti-e-s de différents métiers, les programmes d'échange de jeunes suivant la même formation ont fait leur preuve. La Fondation Margrit et Ruth Stellmacher souhaitant favoriser les échanges entre assistantes médicales en formation, elle s'est engagée à collaborer avec l'organisation «Visite - les apprenti-e-s rendent visite à d'autres apprenti-e-s». L'association «Visite» a été créée par le club Rotary qui s'occupera de l'organisation et du logement des apprenti-e-s. La Fondation Margrit et Ruth Stellmacher remboursera sur demande les frais d'organisation.

die Organisation wird ständig erweitert. «Visite» organisiert für den Austausch Unterkunft und Lehrbetrieb. Natürlich ist die Organisation auf ein genügendes Angebot von Austauschlehrstellen angewiesen.

Die Margrit und Ruth Stellmacher-Stiftung übernimmt die Kosten für Austauschlehrlinge (exkl. Lohn), die von «Visite» plaziert werden.

\section{Durchführung}

Idealerweise kann ein direkter Austausch mit einer Lehrpraxis organisiert werden, die einen Lehrling im selben Lehrjahr beschäftigt. Jeder Lehrling kann selbstverständlich aus eigener Initiative eine Austauschpraxis suchen. Läuft die Abwicklung der Organisation anschliessend über «Visite», werden die Organisationskosten von der Margrit und Ruth Stellmacher-Stiftung 


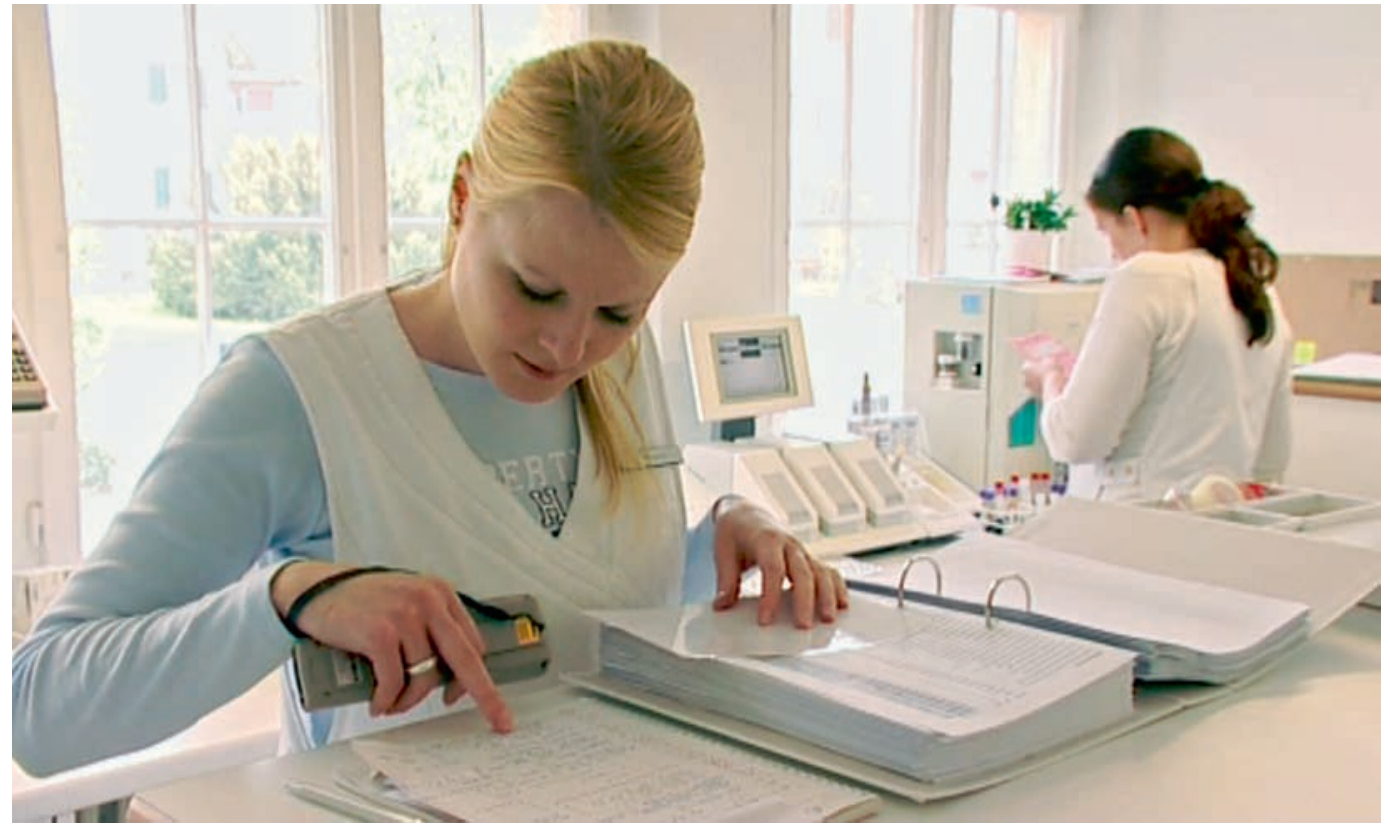

Photo aus dem im Herbst 2007 neu erscheinenden Film über den Beruf der MPA.

(c) Peter Tschudi, Urdorf

in Höhe von bis zu Fr. 1900.- (inkl. Reiseanteil) übernommen. (Die Versicherung ist Sache der Teilnehmerinnen.)

Natürlich muss ein Austauschprojekt immer im Einverständnis mit dem Lehrmeister erfolgen und auf die betrieblichen Gegebenheiten Rücksicht nehmen.

Um genügend Lehrlingen ein Austauschpraktikum zu ermöglichen, benötigen wir genügend Arztpraxen im In- und Ausland, die sich für ein derartiges Projekt zur Verfügung stellen. Selbstverständlich könnte eine Arztpraxis einen Austauschlehrling aufnehmen, auch wenn sie selber keine eigenen Lehrlinge beschäftigt.

Falls sich ausserhalb der Rotariervereinigung «Visite» Gastfamilien zur Verfügung stellen möchten, ist diese Bereitschaft zur Aufnahme eines Austauschlehrlings ebenfalls herzlich willkommen.

Interessierte melden sich bei: sekretariat@ visite.ch, Tel. 0434669915.

\section{Ergebnisse}

Wenn wir bereits im laufenden Jahr genügend Austauschplätze rekrutieren können und mit der Organisation «Visite» hoffentlich genügend Gastfamilien zur Verfügung haben werden, könnten wir mit diesem Projekt rasch beginnen und bereits innert Jahresfrist über die ersten Erfahrungen berichten. 